\title{
Exploring the latent trait of opioid use disorder criteria among frequent nonmedical prescription opioid users
}

\author{
João Mauricio Castaldelli-Maia a, b, c, Laura H. Andrade a, Katherine M. Keyes ${ }^{\mathrm{d}}$, \\ Magdalena Cerdá ${ }^{\text {, }}$ Daniel J. Pilowsky ${ }^{\mathrm{d}, \mathrm{e}}$, Silvia S. Martins ${ }^{\mathrm{d}, *}$ \\ ${ }^{a}$ Section of Psychiatric Epidemiology - LIM 23, Department and Institute of Psychiatry, University of São Paulo Medical School, São Paulo, 05403, Brazil \\ ${ }^{\mathrm{b}}$ Interdisciplinary Group of Studies on Alcohol and Drugs, Department and Institute of Psychiatry, University of São Paulo Medical School, São Paulo, 05403, \\ Brazil \\ ${ }^{c}$ Department of Neuroscience, Medical School, Fundação do ABC, Santo André, SP, 09060, Brazil \\ d Department of Epidemiology, Columbia University Mailman School of Public Health, New York, NY, 10032, USA \\ e New York State Psychiatric Institute, New York, NY, USA \\ ${ }^{\mathrm{f}}$ Department of Emergency Medicine and Violence Prevention Program, University of California, Davis, USA
}

\section{A R T I C L E I N F O}

\section{Article history:}

Received 18 August 2015

Received in revised form

24 March 2016

Accepted 9 May 2016

\section{Keywords:}

Prescription opioids

NMUPO

Doctor shopping

Tolerance

Time spent

\begin{abstract}
A B S T R A C T
Background: There is a need to explore the dimensional and categorical phenotypes of criteria of opioid use disorder among frequent nonmedical users of prescription opioids (NMUPO) users.

Methods: We used pooled data of 2011-2012 National Survey on Drug Use and Health to examine reliability and phenotypic variability in the diagnosis of OUD secondary to NMUPO in a nationallyrepresentative sample of $18+$ years-old frequent past-year NMUPO users $(120+$ days, $n=806$ ). Through exploratory factor analysis (EFA) and latent class analysis (LCA), we examined 10 past-year OUD criteria. We examined associations between the latent classes and sociodemographic/psychiatric/ NMUPO correlates.

Results: OUD criteria were unidimensional, and a three-class model was the overall best fitting solution for characterizing individuals into phenotypes along this unidimensional continuum: a "non-symptomatic class" (40.7\%), "Tolerance-Time spent class" (29.0\%) with high probability of endorsing Tolerance/ Time Spent criteria, and a "High-moderate symptomatic class" (30.1\%). The last class was significantly associated with being male, having insurance and obtaining prescription opioids (PO) nonmedically via "doctor shopping" as compared to the non-symptomatic class. "Tolerance-Time spent class" was significantly associated with being younger (18-25 years) and obtaining PO nonmedically from family/ friends as compared to the non-symptomatic class.

Conclusion: This study revealed the different characteristics and routes of access to PO of different classes of frequent NMUPO users. It is possible that these groups may respond to different interventions, however such conclusions would require a clinical study.
\end{abstract}

(C) 2016 Elsevier Ltd. All rights reserved.

\section{Introduction}

In 2014, there was a higher number of opioid overdoses deaths than in any year on record (CDC, 2015). Overdoses from prescription opioid pain relievers play the main role in this increase in opioid overdose deaths - at least half of these deaths involve a

\footnotetext{
* Corresponding author. Associate Professor of Epidemiology, Department of Epidemiology, Mailman School Of Public Health, Columbia University, 722 West 168th Street, Rm. 509, New York, NY, 10032, USA.

E-mail address: ssm2183@cumc.columbia.edu (S.S. Martins).
}

prescription opioid (CDC, 2015). Since 1999, U.S. prescription opioids sold nearly quadrupled (CDC, 2011). Despite the evident importance of opioid misuse in public health, few studies have examined the unidimensional diagnostic structure of Opioid Use Disorders (OUD) through factor analysis (FA) and item response theory (IRT) (Boscarino et al., 2011; Saha et al., 2012). Even fewer studies have focused on the diagnostic structure as it applies specifically to non-medical use of prescription opioids (NMUPO) (Wu et al., 2009, 2011). FA is a technique that allows reduction of a large number of interrelated variables, such as the DSM criteria (APA, 2013) for OUD, to a smaller number of hidden dimensions (or 
dormant), such as the unidimensional model proposed for OUD according to DSM-5 (APA, 2013). In addition, IRT provides mathematical models for the dimensional structure, proposing forms of representing how individuals fulfill each DSM-5 OUD criteria.

Considering that OUD can be a clinically heterogeneous syndrome (Kendler et al., 2013), studies have also used latent class analysis (LCA) to better understand phenotypic differences of DSMIV OUD criteria among NMUPO (Ghandour et al., 2008; Wu et al., 2011). LCA generates categorical phenotypes (individuals subgroups, subtypes or clusters) based on the response pattern likelihood and is in line with the identification of categorical diagnosis within the unidimensional OUD structure. In the case of DSM-IV criteria for Opioid Dependence for NMUPO, there is a certain degree of uncertainty on which should be the best LCA model ( 2 or 4 classes) (Wu et al., 2011; Ghandour et al., 2008). A reliable model should be repeated in different samples of the general population of the same country. Therefore, it is important to continue investigating this matter in more recent samples. This is even more important nowadays, considering the new edition of DSM (DSM-5), which created a new categorization of symptoms that one needs to report to be classified as having an OUD (APA, 2013).

To date, no study has yet examined phenotypic variation in OUD criteria among frequent NMUPO users. Frequent NMUPO users are of interest because they reflect more accurately the experiences of individuals at high risk of becoming dependent and those already dependent. These frequent users also have a more active role in obtaining pharmaceutical opioids compared to infrequent users (Daniulaityte et al., 2014). In addition, between 2002 and 2010, the rate of NMUPO increased significantly in U.S., paralleling with increases in treatment admissions, overdose deaths, and other negative effects associated with NUMPO in the same period (Paulozzi et al., 2011; Jones, 2012). Examining the categorical phenotypes of OUD among frequent NMUPO users can provide insight into potential subtypes of the most problematic NMUPO users.

Knowing about the potential OUD subtypes (classes) among NMUPO, we gain the ability to identify groups of users that: have different socio-demographic correlates; seek for PO in different ways (i.e., doctor shopping); have different evolution over time and different clinical and psychiatric comorbidities; who respond and search differently to treatment available; among other characteristics of interest. For instance, knowing which are the subgroups that engage in doctor shopping is extremely useful information for practitioners and researchers. Doctor shopping is when someone consults different doctors obtaining overlapping prescriptions (Nordmann et al., 2013), and is considered one of the main means of diversion for prescription medications in the United States (Lineberry and Bostwick, 2004; Pradel et al., 2004).

Through confirmatory FA and IRT, we confirmed and explored the unidimensional model of OUD for a general population sample of frequent NMUPO users in the US. To do this, we generated a proxy of DSM-5 criteria. In addition, we use LCA to explore categorical phenotypes within the best dimensional model using 10 criteria from DSM-5 OUD. We further examine the sociodemographic, psychiatric and NMUPO sources (i.e., doctor-shopping, family/friends) correlates of membership in each latent class, comorbidity of each latent class with other types of substance use disorders, and treatment-seeking behavior.

\section{Material and methods}

\subsection{Sample}

In 2011-2012, the National Survey of Drug Use and Health (NSDUH) collected data from 138,418 respondents [n
$(2011)=70,109 ; \mathrm{n}(2012)=68,309]$ aged 12 or older and was designed to obtain representative samples from the 50 States and the District of Columbia (SAMHSA, 2012, 2013). Two consecutive NSDUH years were combined in order to increase the sample size of frequent NMUPO users (please see more details on Supplementary File 6).

\subsection{Measures}

The NSDUH questionnaire has reliability for substance use in the past-year and lifetime ranging from 0.72 to 0.93 and 0.71 to 0.95 , respectively (Jordan et al., 2008; National Household Survey on Drug Abuse, 2010a,b, 2012, 2013). For past-year and lifetime NMUPO, kappa coefficient is respectively 0.73 and 0.78 . As with all self-report surveys, the validity of responses to questions on substance use and diagnostic symptoms of SUD may be biased. Jordan et al. (2008) have conducted a study to investigate the clinical validity of SUD symptoms in the NSDUH using the Structured Clinical Interview for DSM-IV (SCID-IV) for adults and the Pittsburgh Adolescent Alcohol Research Center's Structured Clinical Interview (PAARC-SCID) for adolescents. Their study showed the level of agreement between the NSDUH and the SCID/PAARC-SCID interviews to be fair to moderate overall. There was somewhat better agreement for dependence than for abuse and for adults than for adolescents (CBHSQ, 2014).

\subsubsection{Past-year frequent NMUPO users}

NMUPO was defined as any self-reported use of prescription pain relievers that were not prescribed for the respondent or that the respondent took only for the experience or feeling they caused (National Household Survey on Drug Abuse, 2010a,b, 2012, 2013). In order to reduce false-positive responses, all respondents were given the following instructions: "These questions are about prescription pain reliever use. We are not interested in your use of over-the-counter pain relievers that can be bought in stores without a doctor's prescription." The survey used discrete questions and a card with pictures of many types of prescription opioids. The respondents were asked which ones he/she had used, as well as frequency of use. Users were classified both by whether they had ever used opioids non-medically and whether they had used in the past year. We defined as past-year frequent NMUPO users those that had used prescription opioids non-medically on at least 120 days in the past-year $(n=806)$. Because there is no standard definition for frequent NMUPO use, our definition of frequent users is similar to the definition existing literature proposed by Jones (2012) to explore different DSM phenotypes among those with a high likelihood of developing OUD (i.e., using POs nonmedically at least twice a week).

The 806 subjects who reported NMUPO at least 120 days in the past year were asked a series of 17 structured questions derived from past-year DSM-IV opioid abuse/dependence criteria embedded in the 2011-2012 NSDUH questionnaire. These questions were combined to generate 10 dichotomous variables matching 10 of the 11 DSM-5 criteria. The only DSM-5 OUD criterion that was not included in the 2011-2012 NSDUH questionnaire was the craving criterion, which is derived from ICD-10 diagnosis of dependence. The abbreviation of the variable names was based on previously published studies (Hasin and Beseler, 2009; Shmulewitz et al., 2010; Castaldelli-Maia et al., 2014a,b): Tolerance, Withdrawal, Larger/Longer, Quit/Control, Time Spent, Given Up, Physical/Psychological, Major Role, Social/Interpersonal, and Hazardous Use.

\subsubsection{Sociodemographic correlates}

The following sociodemographic correlates were included in the regression models based on a previous LCA study (Ghandour et al., 
2008): sex (female and male), age (categorized as 12-17, 18-25, $26-34$, and $\geq 35$ years); education (low: $=$ less than high school; low-average: = high school graduate; high-average: = some college; high: = college graduate); race/ethnicity (non-Hispanic $(\mathrm{NH})$ White, NH Black, Hispanic, and other); marital status (married/ cohabiting, previously married: = separated/divorced/widowed, never married); and income, defined in categories (poverty, low income $=$ up to twice the Federal poverty threshold, high income: = more than twice the Federal poverty threshold, students: $=$ persons aged 18 to 22 living in a College dorm). We also included three variables that were described as important correlates of NMUPO use in non-LCA studies (Nielsen et al., 2011; Fibbi et al., 2012; Wang et al., 2013): Metropolitan Statistical Area (large metropolitan area, small metropolitan area, and nonmetropolitan area); health insurance (yes or no); and employment status (employed, unemployed, and missing).

\subsubsection{Psychiatric comorbidity, NMUPO sources, and other substance dependence}

Three potential psychiatric-related correlates were included in the regression model: (i) past-year anxiety disorder; (ii) past-year major depressive episode (MDE); and (iii) antisocial behaviors. Three variables that indicated sources of NMUPO (how respondents obtained POs in the past month in the last time they used these drugs) were included in the regression model: (i) Doctor shopping; (ii) Bought from a drug dealer, and (iii) Obtained PO from family/ friends. More details on psychiatric comorbidity, NMUPO sources, and other substance dependence can be found in Supplementary File 6.

\subsubsection{Lifetime treatment for prescription opioid disorders and mental health/addiction treatment}

A respondent was classified as receiving past-year mental health or addiction treatment if she/he received treatment in any location for mental health disorders, or alcohol or drug problems, in the past year. Finally, we created a past-year prescription opioid treatment variable based on the self-report data of receiving treatment in the last 12 months, including current treatment (or counseling) for PO use.

\subsection{Statistical analysis}

All analyses were performed with Mplus version 7, using sampling weights and the complex survey design measures. Descriptive statistics were used to describe the sample. Specifically, counts and percentages were used to describe categorical variables.

A confirmatory factor analysis (CFA) unidimensional model was carried out using considering the 10 criteria as categorical variables. IRT models were used to study the latent opioid use disorder construct. IRT models analyze the relationship between the probabilities of an individual to endorse a certain response to an item. IRT analysis implemented in Mplus was used to derive two main parameters, the threshold and the discrimination parameters. The first refers to the 'severity' of a criterion (threshold), with high severity items being those that the individual should have worse OUD (the latent trait) in order to endorse the criterion. The second parameter measures the ability of a criterion to discriminate respondents from low to high levels of the disorder continuum (slope).

LCA was conducted using maximum likelihood ratio estimation. The random option in Mplus was used to ensure convergence of the most successful LCA models. Specifically, 500 sets of random starting values were used in the initial phase, and 5 optimizations were used in the final stage of convergence. This process ensured that the best log-likelihood value for each model was replicated several times. Several statistical indices were used to assess the fit of the models, including log-likelihood (LL), Akaike Information Criterion (AIC), Bayesian Information Criterion (BIC) and samplesize-adjusted BIC (SSABIC). A low value of log-likelihood together with smaller amounts in AIC BIC, and SSABIC may reflect a more parsimonious model (Muthén, 2006). However, the BIC value has been shown to be more reliable than other information (Nylund et al., 2007).

Once the number of classes was determined, we used 2 polytomous logistic regression models (pseudo-class draw method) to investigate the association between latent classes and: (i) NMUPO sources; (ii) sociodemographic characteristics; and (iv) psychiatric correlates with adjustment for sociodemographic characteristics. The Mplus auxiliary option was used to identify the prevalence of alcohol, tobacco, cannabis, cocaine, stimulant, tranquilizer and pain relievers dependence, mental health/addiction treatment and treatment for pain relievers disorders, for which the equality of means across latent classes were tested using pseudo-class-based multiple imputations (Asparouhov, 2007). Chi-squared tests were used to compare the prevalence of these variables among the latent classes.

\section{Results}

Table 1A presents the results of CFA and IRT models. The onedimensional (one-factor model) obtained high loadings $(>0.7)$ on all 10 criteria of OUD among past-year frequent NMUPO. In the IRT models, the criteria with the lowest value on severity were Tolerance and Time Spent. Moreover, the criteria with greater severity in these models were Quit/Control and Hazardous Use. Other criteria that had greater severity were Neglect Roles and Larger/Longer. Regarding discrimination, all the criteria had strong values.

Table $1 \mathrm{~B}$ presents the LCA model fit indices. The best LCA model was the 4-class model. However, one of the latent classes ("Nonloss control class") was too small to investigate correlates of NMUPO ( $<8 \%$ of the sample), which is an aim of the present study. The LCA graph for this model is presented in Supplementary File 2. Based on the LCA index, the second best model was the 3-class model, which had acceptable latent classes sizes. Latent class structure did not differ by age: the 3-class model was the best fit when including the adolescent subsample (Supplementary File 3 and 4).

Table 2 presents the weighted probability of endorsement of OUD criteria in the best-fit model, and the probability of reporting OUD criteria and prevalence for each latent class. The "Nonsymptomatic class" included individuals with probability close to zero for all OUD criteria. The "Tolerance-Time Spent class" included individuals with low probabilities $(<30 \%)$ of meeting all diagnostic criteria, with the exception of a high probability of endorsement of the Tolerance and Time Spent criteria (above 80\%), Withdrawal (48\%) and Physical/Psychological (35\%). Individuals in the "highmoderate symptomatic class" had high $(>70 \%)$ or moderate $(>40 \%)$ probabilities of endorsement all the criteria. The largest class was the "Non-symptomatic class" (40.7\%), followed by the "ToleranceTime Spent class" $(29.0 \%)$ and the "High-moderate symptomatic class" (30.1\%). Approximately half of the individuals who reported Tolerance and Time Spent criteria were in the "Tolerance-Time Spent control class". However, most of the individuals who reported all other criteria were in the "high-moderate symptomatic class". Tolerance was the criteria with highest prevalence (59.7\%) in the total sample of frequent NMUPO, followed by Time Spent (55.6\%). Supplementary File 1 presents the weighted probability of endorsement of OUD criteria in the best-fit model.

Table 3A presents the results of the logistic regression models examining sociodemographic correlates of the two most 
Table 1A

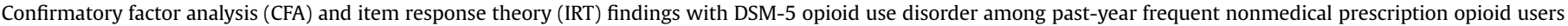
(those that used at least 120 days) in the United States, 2011-2012.

\begin{tabular}{|c|c|c|c|c|c|}
\hline & \multirow[t]{2}{*}{$\mathrm{n}^{*}$} & \multirow[t]{2}{*}{$\%$} & \multirow{2}{*}{$\frac{\mathrm{CFA}}{\text { One factor }}$} & \multicolumn{2}{|l|}{ IRT } \\
\hline & & & & Discrimination (SE) & Severity (SE) \\
\hline Tolerance & 513 & 63.6 & 0.911 & $2.25(0.29)$ & $-0.38(0.05)$ \\
\hline Withdrawal & 314 & 39.0 & 0.743 & $1.11(0.09)$ & $0.37(0.06)$ \\
\hline Larger/longer & 231 & 28.7 & 0.827 & $1.48(0.12)$ & $0.67(0.06)$ \\
\hline Quit/control & 190 & 23.6 & 0.710 & $1.01(0.08)$ & $1.01(0.08)$ \\
\hline Time spent & 485 & 60.2 & 0.918 & $2.35(0.30)$ & $-0.28(0.04)$ \\
\hline Given up & 250 & 31.0 & 0.948 & $3.05(0.36)$ & $0.52(0.05)$ \\
\hline Physical/psychological & 293 & 36.4 & 0.882 & $1.89(0.16)$ & $0.39(0.05)$ \\
\hline Neglect roles & 203 & 25.2 & 0.934 & $2.66(0.31)$ & $0.71(0.05)$ \\
\hline Social/interpersonal & 253 & 31.4 & 0.887 & $1.94(0.16)$ & $0.54(0.05)$ \\
\hline Hazardous & 189 & 23.4 & 0.808 & $1.38(0.11)$ & $0.89(0.06)$ \\
\hline
\end{tabular}

* Estimated value of $\mathrm{n}$ since after de imputation of weights/clusters/strata it is not possible to calculate the exact value of $\mathrm{n}$.

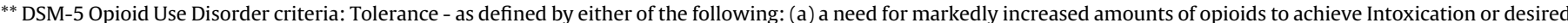

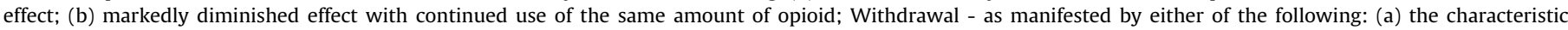

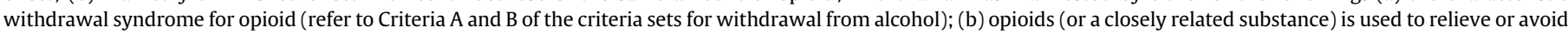

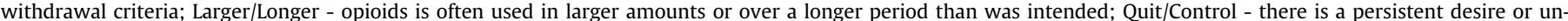

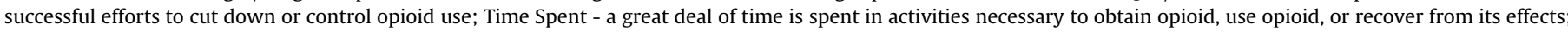

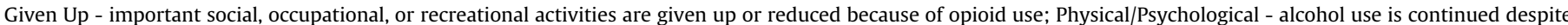

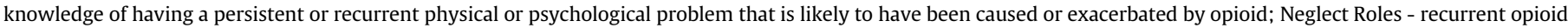

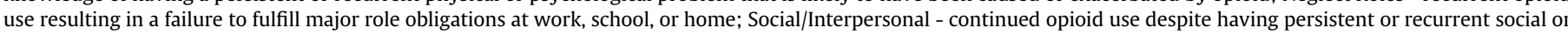

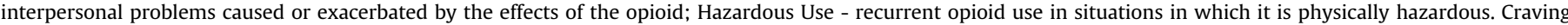
criterion was not included in the present study.

Table 1B

LCA model fit statistics of 10 of the 11 DSM-5 opioid use disorder criteria among those that used prescripton opioids at least 120 days in the past year.

\begin{tabular}{|c|c|c|c|c|c|c|c|c|}
\hline Model & LL & AIC & BIC & SSABIC & $p$-Vuong & Entropy & $\mathrm{df}$ & Low class $\%$ \\
\hline Two-class & -3420.307 & 6930.614 & 7141.758 & 6998.857 & 0.046 & 0.931 & 1001 & 43.5 \\
\hline Three-class & -3103.551 & 6367.102 & 6742.469 & 6488.423 & 0.715 & 0.921 & 990 & 29.0 \\
\hline Four-class & -2956.643 & 6143.286 & 6682.875 & 6317.684 & 0.799 & 0.943 & 975 & 7.4 \\
\hline Five-class & -2878.842 & 6057.684 & 6761.497 & 6285.161 & 0.760 & 0.953 & 965 & 7.3 \\
\hline Six-class & -2760.087 & 5890.174 & 6758.209 & 6170.728 & 0.761 & 0.966 & 954 & 8.4 \\
\hline Seven-class & -2734.234 & 5908.469 & 6940.727 & 6242.100 & 0.755 & 0.967 & 943 & 3.2 \\
\hline Eight-class & -2693.464 & 5896.928 & 7093.410 & 6283.638 & 0.760 & 0.976 & 935 & 1.8 \\
\hline
\end{tabular}

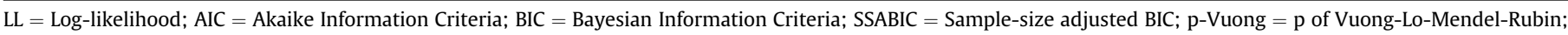
$\mathrm{df}=$ degrees of freedom; $\mathrm{NC}=$ The model did not reach convergence.

Table 2

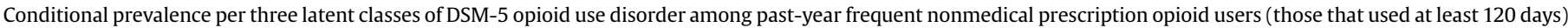
in the United States, 2011-2012.

\begin{tabular}{|c|c|c|c|c|c|c|c|c|c|c|c|}
\hline \multirow[t]{2}{*}{ Criteria } & \multicolumn{3}{|c|}{ “Non-symptomatic class" } & \multicolumn{3}{|c|}{ "Tolerance-time spent class" } & \multicolumn{3}{|c|}{$\begin{array}{l}\text { "High-moderate symptomatic } \\
\text { class" }\end{array}$} & \multicolumn{2}{|l|}{ Total } \\
\hline & $\mathrm{n}^{*}$ & $\%$ & SE & $\mathrm{n}^{*}$ & $\%$ & SE & $\mathrm{n}^{*}$ & $\%$ & SE & $\mathrm{n}^{*}$ & $\%$ \\
\hline Tolerance & 59 & 13.8 & 0.02 & 301 & 89.0 & 0.02 & 242 & 97.0 & 0.01 & 601 & 59.4 \\
\hline Withdrawal & 22 & 5.3 & 0.01 & 147 & 43.6 & 0.03 & 187 & 74.9 & 0.03 & 356 & 35.2 \\
\hline Larger/longer & 5 & 1.1 & 0.01 & 94 & 27.9 & 0.03 & 154 & 61.8 & 0.03 & 253 & 25.0 \\
\hline Quit/control & 11 & 2.6 & 0.01 & 66 & 19.4 & 0.03 & 125 & 50.3 & 0.03 & 202 & 20.0 \\
\hline Time spent & 33 & 7.9 & 0.02 & 298 & 88.1 & 0.02 & 238 & 95.5 & 0.02 & 569 & 56.3 \\
\hline Activities given up & 4 & 0.9 & 0.01 & 51 & 15.1 & 0.03 & 238 & 95.4 & 0.02 & 292 & 28.9 \\
\hline Physical/psychological & 4 & 0.9 & 0.01 & 108 & 32.1 & 0.03 & 220 & 88.3 & 0.02 & 332 & 32.9 \\
\hline Neglect roles & 10 & 2.3 & 0.01 & 22 & 6.6 & 0.02 & 211 & 84.7 & 0.03 & 243 & 24.0 \\
\hline Social/interpersonal & 3 & 0.8 & 0.01 & 72 & 21.2 & 0.03 & 216 & 86.7 & 0.02 & 291 & 28.8 \\
\hline Hazardous use & 0 & 0.0 & 0.00 & 62 & 18.3 & 0.02 & 165 & 66.4 & 0.03 & 227 & 22.5 \\
\hline Total & 424 & 41.93 & 0.00 & 338 & 33.43 & 0.00 & 249 & 24.62 & 0.00 & 1011 & 100.00 \\
\hline
\end{tabular}

* Estimated value of $\mathrm{n}$ since after de imputation of weights/clusters/strata it is not possible to calculate the exact value of $\mathrm{n}$.

symptomatic classes, with the "Non-symptomatic class" as reference. Those in the "Tolerance-Time Spent class" were more likely than those in class the "Non-symptomatic class" to be younger (18-25 years) and Non-Hispanic White (vs. 'Other' racial/ethnic groups) while those in the "High-Moderate symptomatic class" were more likely than those in the "Non-symptomatic class" to be male, Non-Hispanic White (vs. 'Other' racial/ethnic groups) and to have insurance.
Table 3B presents the results of the logistic regression models examining psychiatric and sources correlates, with adjustment for all sociodemographics included in Table 3A. The two symptomatic classes (as compared to class the "Non-symptomatic class") were significantly associated with antisocial behaviors and buying POs from drug dealers in the past month as compared to the nonsymptomatic class. Those in the "Tolerance-Time Spent class" were more likely than those in the "Non-symptomatic class" to 
Table 3A

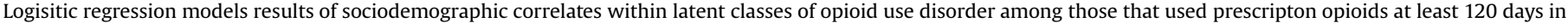
the past year.

\begin{tabular}{|c|c|c|c|c|c|c|c|c|c|c|c|c|}
\hline \multirow[t]{2}{*}{ Variable } & \multicolumn{2}{|c|}{$\begin{array}{l}\text { "Non- } \\
\text { symptomatic" }\end{array}$} & \multicolumn{5}{|c|}{ "Non-symptomatic" } & \multicolumn{5}{|c|}{ "High-moderate symptomatic class" } \\
\hline & $\mathrm{n}^{*}$ & $\%$ & $\mathrm{n}^{*}$ & $\%$ & $\mathrm{aOR}$ & $95 \% \mathrm{CI}$ & $p$ & $\mathrm{n}^{*}$ & $\%$ & aOR & $95 \% \mathrm{CI}$ & $\mathrm{p}$ \\
\hline \multicolumn{13}{|l|}{ Gender } \\
\hline Female & 185 & 43.6 & 112 & 33.0 & 1.00 & & & 72 & 28.8 & 1.00 & & \\
\hline Male & 239 & 56.4 & 226 & 67.0 & 1.64 & $0.89-3.00$ & 0.107 & 177 & 71.2 & 1.51 & $1.25-3.98$ & 0.006 \\
\hline \multicolumn{13}{|l|}{ Income } \\
\hline Medium income & 121 & 28.6 & 93 & 27.6 & 1.00 & & & 65 & 26.3 & 1.00 & & \\
\hline Poverty & 114 & 26.8 & 81 & 23.9 & 1.37 & $0.50-3.71$ & 0.532 & 61 & 24.5 & 1.09 & $0.45-2.64$ & 0.835 \\
\hline High income & 189 & 44.6 & 164 & 48.5 & 1.18 & $0.43-3.24$ & 0.739 & 123 & 49.2 & 1.63 & $0.74-3.57$ & 0.221 \\
\hline \multicolumn{13}{|l|}{ Metropolitan area } \\
\hline Large & 224 & 52.8 & 150 & 44.3 & 1.00 & & & 127 & 50,7 & 1.00 & & \\
\hline Small & 127 & 30.0 & 115 & 34.1 & 1.08 & $0.55-2.13$ & 0.810 & 82 & 33.1 & 0.93 & $0.43-1.96$ & 0.849 \\
\hline Non-metropolitan area & 73 & 17.2 & 73 & 21.6 & 1.45 & $0.63-3.35$ & 0.375 & 40 & 16.2 & 0.99 & $0.38-2.59$ & 0.992 \\
\hline \multicolumn{13}{|l|}{ Insurance } \\
\hline No & 146 & 34.4 & 139 & 41.2 & 1.00 & & & 92 & 37.1 & 1.00 & & \\
\hline Yes & 278 & 65.6 & 199 & 58.8 & 1.10 & $0.62-1.94$ & 0.732 & 157 & 62.9 & 2.08 & $1.11-3.87$ & 0.021 \\
\hline \multicolumn{13}{|l|}{ Employment status } \\
\hline Employed & 259 & 61.0 & 222 & 65.7 & 1.00 & & & 138 & 55.3 & 1.00 & & \\
\hline Unemployment & 165 & 39.0 & 116 & 34.3 & 0.70 & $0.39-1.26$ & 0.242 & 111 & 44.7 & 1.51 & $0.79-2.89$ & 0.210 \\
\hline \multicolumn{13}{|l|}{ Race } \\
\hline Non-hispanic white & 270 & 63.6 & 274 & 81.2 & 1.00 & & & 216 & 86.7 & 1.00 & & \\
\hline Non-hispanic black & 60 & 14.2 & 21 & 6.2 & 0.63 & $0.20-1.90$ & 0.413 & 7 & 2.9 & 0.28 & $0.05-1.35$ & 0.114 \\
\hline Hispanic & 67 & 15.9 & 30 & 8.8 & 0.63 & $0.26-1.48$ & 0.294 & 19 & 7.6 & 0.41 & $0.17-0.96$ & 0.040 \\
\hline Other & 27 & 6.3 & 13 & 3.8 & 0.29 & $0.15-0.58$ & $<\mathbf{0 . 0 0 1}$ & 7 & 2.8 & 0.30 & $0.09-0.92$ & 0.037 \\
\hline \multicolumn{13}{|l|}{ Education (in years) } \\
\hline Low & 107 & 25.3 & 74 & 21.7 & 1.00 & & & 67 & 26.8 & 1.00 & & \\
\hline Low-average & 156 & 36.8 & 130 & 38.6 & 1.45 & $0.57-3.68$ & 0.424 & 82 & 32.8 & 0.67 & $0.28-1.60$ & 0.367 \\
\hline High-average & 124 & 29.2 & 94 & 27.8 & 1.26 & $0.53-3.02$ & 0.596 & 81 & 32.7 & 0.92 & $0.38-2.20$ & 0.861 \\
\hline High & 37 & 8.7 & 40 & 11.9 & 2.65 & $0.79-8.90$ & 0.114 & 19 & 7.7 & 0.87 & $0.16-4.62$ & 0.872 \\
\hline \multicolumn{13}{|l|}{ Marital status } \\
\hline Never married & 212 & 50.1 & 185 & 54.6 & 1.00 & & & 156 & 62.9 & 1.00 & & \\
\hline Widow/separated/divorced & 70 & 16.4 & 70 & 20.8 & 2.50 & $0.84-7.41$ & 0.096 & 51 & 20.3 & 2.15 & $0.85-5.38$ & 0.102 \\
\hline Married/cohabiting & 142 & 33.5 & 83 & 24.6 & 1.24 & $0.47-3.26$ & 0.660 & 42 & 16.8 & 0.59 & $0.21-1.60$ & 0.302 \\
\hline \multicolumn{13}{|l|}{ Age (in years) } \\
\hline $18-25$ & 19 & 4.4 & 31 & 9.1 & 1.00 & & & 19 & 7.4 & 1.00 & & \\
\hline $26-34$ & 275 & 64.8 & 210 & 62.2 & 0.66 & $0.45-0.99$ & 0.046 & 157 & 63.2 & 0.95 & $0.58-1.55$ & 0.851 \\
\hline 35 or more & 131 & 30.8 & 97 & 28.7 & 0.18 & $0.07-0.48$ & 0.001 & 73 & 29.4 & 0.46 & $0.17-1.24$ & 0.221 \\
\hline \multicolumn{13}{|l|}{ Survey year } \\
\hline 2011 & 216 & 50.9 & 176 & 52.1 & 1.00 & & & 133 & 53.4 & 1.00 & & \\
\hline 2012 & 208 & 49.1 & 162 & 47.9 & 1.08 & $0.55-2.10$ & 0.809 & 116 & 46.6 & 1.09 & $0.60-1.98$ & 0.763 \\
\hline
\end{tabular}

Bold: significant associations $(p<0.05)$.

* Estimated value of $\mathrm{n}$ since after de imputation of weights/clusters/strata it is not possible to calculate the exact value of $\mathrm{n}$.

** Non-symptomatic class was the reference category for other latent classes.

have obtained PO from family/friends while those in the "HighModerate symptomatic class" were more likely than those in the "Non-symptomatic class" to have obtained POs via Doctor shopping.

Table 4 presents progressively higher values ("High-moderate symptomatic class" > "Tolerance-Time Spent class" > "Non-symptomatic class") of conditional prevalence of past-year other substance dependence, and previous or current treatment for $\mathrm{PO}$ disorders and Mental Health/Addiction, in each of the 3 latent classes. Despite a high past-year prevalence of opioid dependence among those on the "Tolerance-Time Spent class" and the "Highmoderate symptomatic class" (63.1\% and 98.6\%, respectively), a very low proportion of respondents in "Tolerance-Time Spent class" sought treatment for opioid disorders secondary to NMUPO (8.2\%), in contrast with those in "high-moderate class", in which $36.4 \%$ sought treatment for opioid disorders secondary to NMUPO. The highest prevalence of comorbidity was found for tobacco dependence, followed by alcohol dependence (70.4\% and $29.4 \%$, respectively, in the "high-moderate symptomatic class" and 60.6\%, 15.9\%, respectively, in the "Tolerance-Time Spent class", respectively). Cocaine dependence $(15.5 \%)$ reached a higher value than cannabis dependence (14.4\%) in "High-moderate symptomatic class" which was not the case of "Tolerance-Time Spent class" (5.9\% and $10.6 \%$, respectively). Supplementary File 5 presents the weighted counts and proportions, and the standard errors for each variable included in the logistic regression models and for latent class conditional prevalence.

\section{Discussion}

The CFA and LCA of an epidemiological household sample of US frequent nonmedical prescription opioid users yielded support for an unidimensional model of OUD criteria and for a three-class model (LCA) as the overall best solution for dividing frequent users in phenotypes along this unidimensional continuum. The three-class model identified a "Non-symptomatic" class, an intermediate class with high probability of endorsing Tolerance and Time Spent symptoms, and a high-moderate symptom class. There was also an increasing prevalence of past-month tobacco dependence, and past-year alcohol dependence from the "Non-symptomatic class" to the "Tolerance-Time spent class" to the "Highmoderate symptomatic class". Interestingly, those in the "Tolerance-Time Spent class" were more likely to obtain PO from family/ friends while those in the "High-moderate symptomatic class" (vs. 
Table 3B

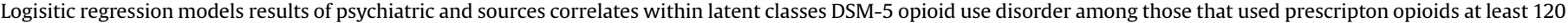
days in the past year.

\begin{tabular}{|c|c|c|c|c|c|c|c|c|c|c|c|c|}
\hline \multirow[t]{2}{*}{ Variable } & \multicolumn{2}{|c|}{$\begin{array}{l}\text { "Non- } \\
\text { symptomatic" }\end{array}$} & \multicolumn{5}{|c|}{ “Tolerance-time spent class” } & \multicolumn{5}{|c|}{$\begin{array}{l}\text { "High-moderate } \\
\text { symptomatic class" }\end{array}$} \\
\hline & $\mathrm{n}^{*}$ & $\%$ & $\mathrm{n}^{*}$ & $\%$ & $\mathrm{aOR}$ & $95 \% \mathrm{CI}$ & $p$ & $\mathrm{n}^{*}$ & $\%$ & $\mathrm{aOR}$ & $95 \% \mathrm{CI}$ & $\mathrm{p}$ \\
\hline Anxiety disorders & 56 & 13.1 & 66 & 19.4 & 1.31 & $0.58-2.98$ & 0.506 & 66 & 26.7 & 1.88 & $0.82-4.29$ & 0.132 \\
\hline $\begin{array}{l}\text { Major depressive } \\
\text { disorder }\end{array}$ & 56 & 13.1 & 62 & 18.4 & 1.44 & $0.68-3.03$ & 0.330 & 65 & 26.0 & 1.61 & $0.65-3.98$ & 0.302 \\
\hline Antisocial behaviors & 51 & 12.0 & 118 & 34.8 & 3.33 & $1.75-6.35$ & $<0.001$ & 150 & 60.2 & 9.43 & $4.53-19.66$ & $<0.001$ \\
\hline Doctor shopping & 81 & 19.0 & 80 & 23.7 & 1.74 & $0.80-3.79$ & 0.162 & 74 & 29.6 & 2.60 & $1.29-5.24$ & 0.008 \\
\hline $\begin{array}{l}\text { Bought from a drug } \\
\text { dealer }\end{array}$ & 28 & 6.7 & 94 & 27.7 & 4.81 & $1.79-12.88$ & 0.002 & 78 & 31.3 & 6.58 & $2.89-14.98$ & $<0.001$ \\
\hline $\begin{array}{l}\text { Obtained PO from } \\
\text { family/friends }\end{array}$ & 137 & 32.4 & 199 & 58.8 & 2.97 & $1.54-5.72$ & 0.001 & 121 & 48.4 & 1.52 & $0.82-2.78$ & 0.176 \\
\hline
\end{tabular}

Bold: significant associations $(p<0.05)$.

* Estimated value of $\mathrm{n}$ since after de imputation of weights/clusters/strata it is not possible to calculate the exact value of $\mathrm{n}$.

** Non-symptomatic class was the reference category for other latent classes.

$\mathrm{PO}=$ prescription opioids

those in the "Non-symptomatic class") were more likely to be doctor shoppers.

Only two studies evaluated the dimensionality of OUD (Wu et al., 2009, 2011) by EFA and both examined the dimensionality of criteria among those any past-year NMUPO. Our study shares some similarities with these studies but focuses on a sample that included only frequent users. Frequent users are a group that is most likely to generate data that could inform prevention and treatment because these individuals are at considerable risk for having OUD. Both studies gave support to an unidimensional model (Wu et al., 2009, 2011), which allowed us to run a CFA in our more restricted sample of frequent users. Our study did not include the Legal Problems criterion because of its exclusion in DSM-5 (American Psychiatric Association, 2013), gives support to a unidimensional model for OUD, despite not including the Craving criterion. An important future direction is to assess LCA in data sources with complete DSM-5 criteria as it was carried out for the entire class of opioids (Tarrahi et al., 2015).

Our IRT model findings are presented in a similar way to the findings of previous studies with U.S. adolescent (Wu et al., 2009) and adult (Wu et al., 2011) samples of any nonmedical users. Larger/ Longer and Quit/Control were found to be the criteria that endorsed by those individuals who probably endorsed several other OUD criteria. In contrast, Tolerance and Time Spent seem to be the first OUD criteria endorsed from the 10 criteria tested (Wu et al., 2009, 2011). Interestingly, Hazardous Use was more severe among individuals in our study than in Wu et al.' study (2011). Wu et al. (2011) divided their sample into most affected and less affected groups by factor mixture model. We compared our IRT results with the most affected group since we focused our analyses on frequent users.

To date, only a few studies have used LCA to understand phenotypic differences of opioid dependence symptoms among nonmedical prescription opioid users and none of them focused on frequent users (Ghandour et al., 2008; Wu et al., 2011). Similarly to these studies (Ghandour et al., 2008; Wu et al., 2011), we also find a large non-symptomatic class and a class with a high probability of satisfying the criteria "Tolerance" and "Time Spent" with a moderate probability of endorsement of the other criteria. In addition, we also found a "high-moderate symptomatic class" as Ghandour et al.'s study (2008). The only class found by Ghandour et al. (2008) and not found in the present study was one latent class related to adolescents. This could be explained by the fact that we did not include adolescents in our analyses. Despite similarities regarding the latent classes found in Ghandour et al.' study and the present study, the sociodemographic correlates were quite different, maybe because we focused on frequent NMUPO users while the Ghandour study focused on any past-year NMUPO. For example, they reported that being female was significantly associated with the symptomatic classes, which differs from our finding of association of males having a higher likelihood of being in the most symptomatic class compared to females. Differences could be explained by the differences between the selected samples (any NMUPO and frequent NMUPO, respectively). We analyzed data from a sample of adults only, with more frequent use, and with data collected about a decade later. Unfortunately, Wu et al. (2011) did not analyze sociodemographic correlates of the latent classes.

Both symptomatic classes did not differ regarding the psychiatric correlates - both were more strongly associated with antisocial behaviors as compared to the "Non-symptomatic class".

Table 4

Conditional prevalence per three latent classes of DSM-5 opioid use disorder among those that used prescripton opioids at least 120 days in the past year.

\begin{tabular}{|c|c|c|c|c|}
\hline \multirow[t]{2}{*}{ Latent class } & \multirow{2}{*}{$\frac{\text { “Non-symptomatic class" }}{\%(\mathrm{SE})}$} & \multirow{2}{*}{$\begin{array}{l}\text { “Tolerance-time spent class" } \\
\%(\mathrm{SE})\end{array}$} & \multirow{2}{*}{$\begin{array}{l}\text { "High-moderate symptomatic class" } \\
\%(\mathrm{SE})\end{array}$} & \multirow{2}{*}{$\begin{array}{l}\text { Chi-squared test } \\
\mathrm{p}\end{array}$} \\
\hline & & & & \\
\hline Treatment for DSM-IV pain relivers (opioid) disorders & $1.1(0.01)$ & $8.2(0.01)$ & $36.4(0.03)$ & $<0.001$ \\
\hline Treatment for mental health/addiction & $25.2(0.02)$ & $36.7(0.03)$ & $58.0(0.03)$ & $<0.001$ \\
\hline DSM-IV alcohol dependence & $15.2(0.02)$ & $15.9(0.02)$ & $29.4(0.03)$ & $<0.001$ \\
\hline Tobacco dependence (FTND or NDSS) & $48.6(0.02)$ & $60.6(0.03)$ & $70.4(0.03)$ & $<0.001$ \\
\hline DSM-IV cannabis dependence & $4.3(0.01)$ & $10.6(0.02)$ & $14.4(0.02)$ & $<0.001$ \\
\hline DSM-IV cocaine dependence & $5.5(0.01)$ & $5.9(0.01)$ & $15.5(0.02)$ & 0.001 \\
\hline DSM-IV stimulant dependence & $0.6(0.01)$ & $3.9(0.01)$ & $11.7(0.02)$ & $<0.001$ \\
\hline DSM-IV tranquilizers dependence & $0.0(0.01)$ & $3.5(0.01)$ & $12.5(0.02)$ & $<0.001$ \\
\hline DSM-IV pain relivers (opioid) dependence & $0.0(0.01)$ & $63.1(0.03)$ & $98.6(0.01)$ & $<0.001$ \\
\hline
\end{tabular}

$\mathrm{SE}=$ Linearized Standard Error. 
However, regarding sources of NMUPO, there was an interesting difference. Despite both symptomatic classes (as compared to the "Non-symptomatic class") being more strongly associated with buying from a drug dealer, the "Time Spent-Tolerance class" was significantly associated with obtaining PO from family/friends, while the "High-Moderate symptomatic class" was significantly associated with "doctor shopping". Most importantly, these differences persisted after adjustment for sociodemographic characteristics, including gender, which has been associated with these types of sources - i.e., female gender is more strongly associated with doctor-shopping (Back et al., 2010). It is important to target respondents from both classes to seek treatment, since despite the prevalence of DSM-IV Opioid Dependence in those classes being high, treatment prevalence is very low in both.

More than $60 \%$ of the individuals in the "high-moderate symptomatic class" did not seek for treatment for OUD. Considering the high prevalence of comorbid conditions, including multiple substance use disorders and a higher lifetime prevalence of mental health/addiction treatment (58\%), it is possible that would be easier to find these individuals, who were predominantly Non-Hispanic White males with health insurance, in psychiatric and substance abuse treatment settings if they seek treatment for comorbid conditions. However, there must be a contingent of individuals in this class who do not seek treatment both for OUD and comorbid conditions.

Due to the cross-sectional design of the NSDUH survey, the associations reported here, even if statistically significant, reflect correlations and should not be viewed as providing evidence supporting etiological pathways. Although the NSDUH is an annually administered national survey, it does not capture the same individuals every year. A second limitation is the use of self-report. However, the use of a computerized reporting system, ACASI, minimizes social desirability biases, and yields data likely to be internally consistent and complete (Turner et al., 1998). We have also not included the Craving criterion in the present analyses since the NSDUH data does not include it, despite some recent studies that state the importance of this symptom for different samples of prescription opioid users (Ashrafioun and Carels, 2014; Wasan et al., 2012). In addition, the definition of doctor shopping used was broad, and has the potential to be misleading for policy makers. Unfortunately, the NSDUH does not have a more restrictive/refined definition of doctor shopping. We also could not distinguish whether these NMUPO users first started using these drugs when legitimately prescribed (e.g., pain relief) or when obtained illegally (i.e., to get high); such data were unavailable in the NSDUH. Gathering such data in future studies will help us better understand the profiles of these users, which may be distinct. Finally, the analytic focus of this manuscript was specifically on frequent NMUPO users. Thus, it was not possible to generalize it to people that less frequently engage NMUPO.

In summary, this study showed that frequent NMUPO users exhibit three different types of OUD phenotypes. Both the Tolerance and Time Spent criteria (which are included in DSM-5 OUD) seem important to identify phenotypes of frequent prescription opioid users in this and in previous studies. There is an intermediate class of Non-Hispanic White young adults who get PO from family and friends, with high probability of endorsing tolerance and spend a lot of time searching for, using or recovering from PO. Finally, the most symptomatic class consisting of White NonHispanic males with health insurance, and that is significantly associated doctor shopping. Both classes call attention because of their low level of treatment seeking. It is possible that these groups may respond to different interventions, however such conclusions would require a clinical study. The present study did not find very different phenotypes of individuals among frequent nonmedical prescription opioid users (i.e., physical versus psychological), but the phenotypes found in this study are within the OUD continuum, which could corroborate with the findings of the several studies evaluating the dimensionality of the DSM-5 Substance Use Disorder (Shmulewitz et al., 2015). However, it is undeniable that both symptomatic classes described in this study have very different socio-demographic correlates, and present an important differentiation in regards to prescription opioids sources (doctor shopping versus family). Therefore, our study presents important public health implications to distinguish subgroups of frequent nonmedical prescription opioid users. Furthermore, this study may be of particular interest for future phenotypic investigations in different populations in the U.S. and in other countries.

\section{Financial support}

- Dr. Castaldelli-Maia receives Pfizer Independent Grant for Learning and Change (IGLC) managed by Global Bridges (Healthcare Alliance for Tobacco Dependence Treatment) hosted at Mayo Clinic, to support free smoking cessation treatment training in addiction/mental health care units in Brazil (grant IGLC 13513957) which had no relationship with the present study.

- Dr. Martins is currently supported by NIH-NICHD R01HD060072, NIH-NIDA R01DA037866 and NIH-NIDA 1R01DA039962.

- Dr. Keyes is currently supported by NIH-NIAAA K01AA021511.

- Dr. Cerdá is currently supported by NIH-NIDA K01DA030449-01 and NIH-NIDA 1R01DA039962.

\section{Contributors}

Authors JMC-M, LHA and SSM designed the study. Authors JMCM, LHA, KMK, MC, DJP and SSM managed the literature searches and summaries of previous related work. JMC-M and SSM undertook the statistical analysis. Author JMC-M and SSM wrote the first draft of the manuscript. All authors contributed to and have approved the final manuscript.

\section{Conflict of interest}

Dr. Martins worked in 2014 as a consultant to Purdue Pharma on secondary data analyses of nonmedical prescription opioid and alcohol use in a different dataset, unrelated to the data described in this manuscript.

\section{Acknowledgements}

- Substance Abuse and Mental Health Data Archive (SAMHDA), sponsored by U.S. Department of Health and Human Services.

- Inter-university Consortium for Political and Social Research (ICPSR), U.S.

- SAMHSA and ICPSR have no responsibility for the use of the data or for the interpretations and inferences made in the present study.

\section{Appendix A. Supplementary data}

Supplementary data related to this article can be found at http:// dx.doi.org/10.1016/j.jpsychires.2016.05.007.

\section{References}

American Psychiatric Association, 2013. Diagnostic and Statistical Manual of Mental 
Disorders, fifth ed. American Psychiatric Association, Arlington.

Ashrafioun, L., Carels, R.A., 2014. Prescription opioid use among university students: assessment of post-cue exposure craving. Addict. Behav. 39, 586-592.

Asparouhov, T., 2007. Wald Test of Mean Equality for Potential Latent Class Predictors in Mixture Modeling. http://www.statmodel.com/download/MeanTest1. pdf (accessed in 13.12.13.).

Back, S.E., Payne, R.L., Simpson, A.N., Brady, K.T., 2010. Gender and prescription opioids: findings from the national survey on drug use and health. Addict. Behav. 35, 1001-1007.

Boscarino, J.A., Rukstalis, M.R., Hoffman, S.N., Han, J.J., Erlich, P.M., Ross, S., Gerhard, G.S., Stewart, W.F., 2011. Prevalence of prescription opioid-use disorder among chronic pain patients: comparison of the DSM-5 vs. DSM-4 diagnostic criteria. J. Addict. Dis. 30, 185-194.

Castaldelli-Maia, J.M., Martins, S.S., de Oliveira, L.G., van Laar, M., de Andrade, A.G., Nicastri, S., 2014a. Use transition between illegal drugs among Brazilian university students. Soc. Psychiatry Psychiatr. Epidemiol. 49, 385-394.

Castaldelli-Maia, J.M., Silveira, C.M., Siu, E.R., Wang, Y.P., Milhorança, I.A., Alexandrino-Silva, C., Borges, G., Viana, M.C., Andrade, A.G., Andrade, L.H., Martins, S.S., 2014b. DSM-5 latent classes of alcohol users in a population-based sample: results from the São Paulo megacity mental health survey, Brazil. Drug Alcohol Depend. 136, 92-99.

Center for Behavioral Health Statistics and Quality, 2014. National Survey on Drug Use and Health (NSDUH): Summary of Methodological Studies, 1971-2014. Substance Abuse and Mental Health Services Administration. MD, Rockville.

Centers for Disease Control and Prevention (CDC), 2011. Vital signs: overdoses of prescription opioid pain relievers-United States, 1999-2008. Morb. Mortal. Wkly. Rep. 60, 1487-1492.

Centers for Disease Control and Prevention (CDC), 2015. Increases in drug and opioid overdose deaths-United States, 2000-2014. Morb. Mortal. Wkly. Rep. 64, $1378-1382$.

Daniulaityte, R., Falck, R., Carlson, R.G., 2014. Sources of pharmaceutical opioids for non-medical use among young adults. J. Psychoact. Drugs 46, 198-207.

Fibbi, M., Silva, K., Johnson, K., Langer, D., Lankenau, S.E., 2012. Denial of prescription opioids among young adults with histories of opioid misuse. Pain Med. 13, 1040-1048.

Ghandour, L.A., Martins, S.S., Chilcoat, H.D., 2008. Understanding the patterns and distribution of opioid analgesic dependence symptoms using a latent empirical approach. Int. J. Methods Psychiatr. Res. 17, 89-103.

Hasin, D.S., Beseler, C.L., 2009. Dimensionality of lifetime alcohol abuse, dependence and binge drinking. Drug Alcohol Depend. 101, 53-61.

Jones, C.M., 2012. Frequency of prescription pain reliever nonmedical use: 2002-2003 and 2009-2010. Arch. Intern. Med. 172, 1265-1267.

Jordan, B.K., Karg, R.S., Batts, K.R., Epstein, J.F., Wiesen, C., 2008. A clinical validation of the national survey on drug use and health assessment of substance use disorders. Addict. Behav. 33, 782-798.

Kendler, K.S., Ohlsson, H., Sundquist, K., Sundquist, J., 2013. A latent class analysis of drug abuse in a national Swedish sample. Psychol. Med. 43, 2169-2178.

Lineberry, T.W., Bostwick, J.M., 2004. Taking the physician out of "physician shopping": a case series of clinical problems associated with Internet purchases of medication. Mayo Clin. Proced. 79, 1031-1034.

Muthén, B.O., 2006. Should substance use disorders be considered as categorical or dimensional? Addiction 101, S6-S16.

National Household Survey on Drug Abuse, 2010a. 2009 public Use File and Codebook - Substance Abuse and Mental Health Services Administration. Office of Applied Studies, Rockville.

National Household Survey on Drug Abuse, 2012. 2011 public Use File and Codebook - Substance Abuse and Mental Health Services Administration. Office of Applied Studies, Rockville.

National Household Survey on Drug Abuse, 2013. 2012 public Use File and Codebook - Substance Abuse and Mental Health Services Administration. Office of Applied Studies, Rockville.
National Survey On Drug Use And Health, 2010b. In: S. A. A. M. H. S (Ed.), Nationa Survey on Drug Use and Health: Overview and Summary of Substate Region Estimation Methodology, 2008-2010-Administration. http://www.samhsa.gov/ data/NSDUH/substate2k10/Methodology/NSDUHsubstateMethodology2010.pdf (accessed in 13.10.13.).

Nielsen, S., Cameron, J. Lee, N., 2011. Characteristics of a nontreatment-seeking sample of over-the-counter codeine users: implications for intervention and prevention. J. Opioid Manag. 7, 363-370.

Nordmann, S., Pradel, V., Lapeyre-Mestre, M., Frauger, E., Pauly, V., Thirion, X. Mallaret, M., Jouanjus, E., Micallef, J., 2013. Doctor shopping reveals geographical variations in opioid abuse. Pain Physician 16 (1), 89-100.

Nylund, K.L., Asparouhov, T., Muthén, B.O., 2007. Deciding on the number of classes in latent class analysis and growth mixture modeling: a Monte Carlo simulation study. Struct. Equ. Model. 14, 535-569.

Paulozzi, L.J., Jones, C., Mack, K., Rudd, R., 2011. Centers for disease control and prevention (CDC). Vital signs: overdoses of prescription opioid pain relievers-United States, 1999-2008. Morb. Mortal. Wkly. Rep. 60, 1487-1492.

Pradel, V., Thirion, X., Ronfle, E., Masut, A., Micallef, J., Bégaud, B., 2004. Assessment of doctor-shopping for high dosage buprenorphine maintenance treatment in a French region: development of a new method for prescription database. Pharmacoepidemiol. Drug Saf. 13, 473-481.

Saha, T.D., Compton, W.M., Chou, S.P., Smith, S., Ruan, W.J., Huang, B., Pickering, R.P. Grant, B.F., 2012. Analyses related to the development of DSM-5 criteria for substance use related disorders: 1. Toward amphetamine, cocaine and prescription drug use disorder continua using item response theory. Drug Alcohol Depend. 122, 38-46.

Shmulewitz, D., Greene, E.R., Hasin, D., 2015. Commonalities and differences across substance use disorders: phenomenological and epidemiological aspects. Alcohol. Clin. Exp. Res. 39 (10), 1878-1900.

Shmulewitz, D., Keyes, K., Beseler, C., Aharonovich, E., Aivadyan, C., Spivak, B. Hasin, D., 2010. The dimensionality of alcohol use disorders: results from Israel. Drug Alcohol Depend. 111, 146-154.

Substance Abuse and Mental Health Services Administration, 2012. Results from the 2011 National Survey on Drug Use and Health: Summary of National Findings. Substance Abuse and Mental Health Services Administration, Rockville, MD, 2012. NSDUH Series H-44, HHS Publication No (SMA): 12-4713.

Substance Abuse and Mental Health Services Administration, 2013. Results from the 2012 National Survey on Drug Use and Health: Summary of National Findings. Substance Abuse and Mental Health Services Administration, Rockville, MD, 2013. NSDUH Series H-46, HHS Publication No. (SMA) 13-4795.

Tarrahi, M.J., Rahimi-Movaghar, A., Zeraati, H., Motevalian, S.A., Amin-Esmaeili, M. Hajebi, A., Sharifi, V., Radgoodarzi, R., Hefazi, M., Fotouhi, A., 2015. Latent class analysis of DSM-5 criteria for opioid use disorders: results from the Iranian National Survey on mental health. Eur. Addict. Res. 21, 144-152.

Turner, C.F., Ku, L., Rogers, S.M., Lindberg, L.D., Pleck, J.H., Sonestein, F.L., 1998 Adolescent sexual behavior, drug use, and violence: increased reporting with computer survey technology. Science 280, 867-873.

Wang, K.H., Becker, W.C., Fiellin, D.A., 2013. Prevalence and correlates for nonmedical use of prescription opioids among urban and rural residents. Drug Alcohol Depend. 127, 156-162.

Wasan, A.D., Ross, E.L., Michna, E., Chibnik, L., Greenfield, S.F., Weiss, R.D. Jamison, R.N., 2012. Craving of prescription opioids in patients with chronic pain: a longitudinal outcomes trial. J. Pain 13, 146-154.

Wu, L.T., Ringwalt, C.L., Yang, C., Reeve, B.B., Pan, J.J., Blazer, D.G., 2009. Construct and differential item functioning in the assessment of prescription opioid use disorders among American adolescents. J. Am. Acad. Child Adolesc. Psychiatry $48,563-572$

Wu, L.T., Woody, G.E., Yang, C., Pan, J.J., Blazer, D.G., 2011. Abuse and dependence on prescription opioids in adults: a mixture categorical and dimensional approach to diagnostic classification. Psychol. Med. 41, 653-664. 